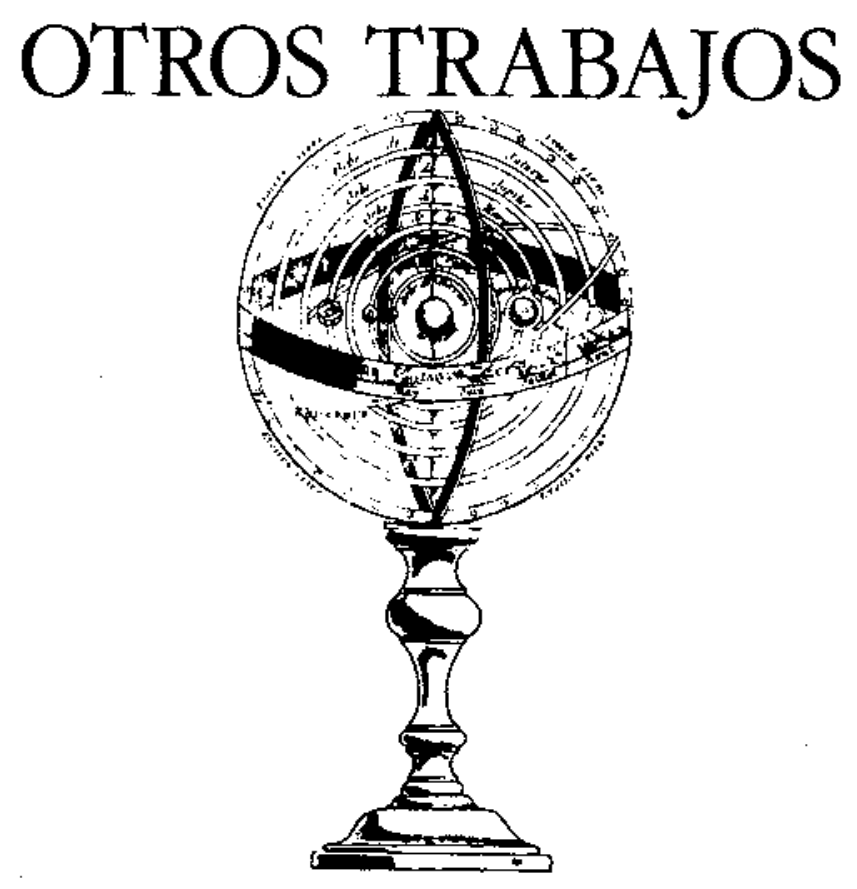

\title{
REFLEXIONES EN TORNO AL CONCEPTO DE ENERGIA. IMPLICACIONES CURRICULARES
}

\author{
SEVILLA SEGURA, $C$.
}

IB «Isabel de Villena», Valencia

\section{SUMMARY}

After an analysis of the difficulty of the energy concept, the paper proposes a review of this subject in the traditional way. in secondary school curricula, in favor of the incorporation of some Thermodynamics concepts. Without these, the energy concept is limited and in this manner conditions its incorporation to the student's cognitive structure by losing a large part of its explanatory power.

\section{INTRODUCCION}

En estos momentos, en que la Reforma de las Enseñanzas Medias promueven el análisis y la reflexion sobre nuestra tantas veces rutinaria tarea, cobran actualidad en nuestro paŕs temas a los que desde ya hace algunos antos dedican atención frecuente las publicaciones especializadas extranjeras. Uno de estos temas, que probablemente se verá potenciado, es el relacionado con la energia y su tratamiento curricular.
Esta reflexión viene motivada por dos tipos de razones. Por una parte, en los actuales libros de texto se introduce el concepto de energía de forma incompleta, parcial, limitándolo - sin advertirlo- a alguno de sus significados, sin relación entre unas manifestaciones y otras, por ejemplo, energia en Mecánica y en Electricidad. Por otra parte, un tema, como el de la energia, con fuertes implicaciones económicas y repercusio- 
nes en la vida cotidiana presenta peculiaridades distintas a aquellos otros cuyo primer contacto se estabiece en la escuela y desarrolla, por tanto, actitudes iniciales en los alumnos distintas. La planificacion del estudio escolar de estos temas no puede pasar por alto esto, y si se pretende realizar un tratamiento riguroso será necesario clarificar aquellos aspectos que en el uso extraescolar puedan aparecer confusos evitando agudizar contradicciones entre la acepción cotidiana del término y la interpretación escolar del mismo.

El problema plantea una doble vertiente, la introducción de los conceptos físicos relacionados con la energía y la necesidad de tomar en consideración las implicaciones derivadas de su repercusión social. El alumno empieza el estudio del tema de energía con bastantes ideas formadas y con inquietudes, con necesidad de encontrar respuestas a cuestiones relacionadas con aspectos de su vida, lo cual no sucede con la mayoria de los otros temas estudiados.

\section{Algunas DIfICULTADES DEL CON- CEPTO DE ENERGIA}

La introducción rigurosa, clara y general de cualquier concepto no suele ser fácil, normalmente. Si además ese concepto es abstracto como el de energia y se encuentra relacionado con otros conceptos también abstractos y con diversidad de significados, la situación se complica, tanto más si pretendemos acercarnos a situaciones reales, que obligan a tener en cuenta la degradación de la energía, introduciéndonos en la Termodinámica.

El tema recibe atención frecuente por parte de distintos investigadores en didáctica, por lo que intentaremos facilitar el camino comentando algunas aportaciones significativas.

Desde el punto de vista didáctico los estudios relativos a los problemas de la energia se enmarcan en tres grandes líneas, relacionadas entre si:

- Concepciones que mantienen los alumnos, formadas previamente al proceso de instrucción escolar. Introducción del concepto de energía.

- La conservación de la energía. Principio de conservación. Degradación de la energía.

- Educación energética.

En la linea sugerida por la primera corriente se inserta un análisis de los conceptos de energía de alumnos de 14 a 18 antos realizado por Watts (1983) que pone de manifiesto la disparidad de nociones que evoca el término de energia en los distintos alumnos. Watts propone una clasificación de respuestas en siete esquemas conceptuales: 1) energía asociada a capacidades humanas; 2) energía como «depósito» que será origen de actividades; 3) la energla como «ingrediente», algo que no está «aimacenado» en un sistema sino que aparece al interactuar con él; 4) la energía como actividad, por ejemplo, el movimiento es energfa; 5 ) la energla como producto de la actividad; 6) energfa funcional, por ejemplo gasolina o cualquier otro tipo de combustible; 7) la energía es un «fluido» que se transfiere de un sistema a otro.

La "definición» de energfa como capacidad para realizar trabajo es frecuentemente contestada por considerar que está limitada al campo de la Mecánica y que entra en contradicción con las leyes de la Termodinámica. Pero, además, resulta ser contradictoria con las mismas explicaciones presuntamente clarificadoras que acompañan a la definición. Así, un cuerpo situado a una altura tiene una cierta energfa potencial (capacidad para realizar trabajo). Esta energia se va transformando a medida que el cuerpo en su descenso gana velocidad. Cuando el cuerpo llega al suelo la energla cinética se convierte en calor y sonido, con lo cual se conserva la energia pero no la capacidad para realizar trabajo (Hicks, 1983).

Algunos autores proponen introducir el concepto de energía en un contexto adecuado, no restringido a la Mecánica como suele hacerse, relacionando siempre el concepto de energia con la conservación. De esta forma pretenden superar algunas confusiones frecuentes en la mente del alumno con otros conceptos relacionados: trabajo, potencia, fuerza. Se sugiere también, dedicar más tiempo a la degradación de la energia que a la propia conservación (Duit, 1984).

El análisis de la contradicción entre el principio de conservación de la energía, tal como habituaimente se enuncia, y las leyes de la Termodinámica pueden dar respuesta a los problemas suscitados cuando el alumno compara lo aprendido en clase - la energia se conserva - con las noticias relativas a la crisis energética y ha levantado voces favorables a la incorporacion de la Termodinámica a los curricula escolares, desde niveles elementales, proponiendo realizar estudios cualitativos sencillos de la energla que tomen como punto de partida los procesos que suceden espontáneamente, haciendo hincapié en que los procesos inversos requieren del uso de dispositivos que consuman combustible, y por tanto, que gasten energla (Haber-Schaim, 1983).

Por lo que respecta a la educación energética, que hace referencia a todos los problemas relacionados con las distintas fuentes de energia, su utilización, racionalización del consumo energético, etc., y sin ánimo de exhaustividad, se proporciona una reseffa bibliográfica de algunas de las publicaciones más recientes, indicativa del nivel de interés con que en otros paises se está abordando el tema.

Las consideraciones anteriores permiten enunciar algunas conclusiones:

1. La complejidad y riqueza del concepto de energfa, así como la imprecisión cometida al definirlo relacio- 
nado exclusivamente con transformaciones mecánicas.

2. La necesidad de proporcionar un marco conceptual suficientemente amplio para incorporar el concepto de energía, de la que deriva la obligatoriedad de introducirlo en diversas situaciones en las que se manifieste de formas distintas.

3. Las posibilidades del principio de conservación para contribuir a la comprensión del concepto de energia, sabiendo en qué condiciones varía, cuándo se transforma pero permanece constante, etc.

4. Las posibilidades que ofrece la incorporación de los conceptos relativos a la crisis energética para mostrar la capacidad explicativa del término energía aun cuando para ello sea necesario introducir algunos conceptos termodinámicos.

\section{CUESTIONES A CONSIDERAR EN UNA PROPUESTA DIDACTICA}

Si tomamos como indicador del tratamiento que mayoritariamente se da en las aulas al tema de la energía, el contenido de los libros de texto en uso, podemos afirmar que responde básicamente al esquema: trabajopotencia-energía-principio de conservación, todo ello sin ningún ejemplo extraño a la Mecánica y por supuesto diferenciado de otro tema que es el de calor.

Además, como los conceptos puestos en juego son complejos, se adopta el camino más cómodo: simplificar. Se procede a definirlos de una manera concisa (y por tanto limitada), en muchas ocasiones mediante una ecuación simplemente.

Bajo una presunta justificación didáctica, en estos planteamientos se ignora toda razón reiacionada con el aprendizaje. No se tiene en cuenta que los alumnos llegan al aula con ideas formadas que no cambiarán fácilmente (Waits, 1983; Duit, 1984; Bliss y Ogborn, 1985). Se olvida también, que la formación de un nuevo concepto, su incorporación a la estructura cognoscitiva, es un largo proceso que requiere una planificación minuciosa en la que se debe considerar tanto la lógica interna del contenido como la forma de acceder a él, y esto presenta rasgos distintos según la edad del aiumno, y su propio desarrolio intelectual (Flavell, 1984; Piaget, 1972). En ningún caso simplificar un concepto contribuirá a su mejor comprensión. Si la mente del alumno no ha alcanzado el grado de desarrollo adecuado, el aprendizaje de ese concepto no será posible y si el desarrollo es pertinente lo dificultará la desvirtuación de la simplificación del contenido. Lo más que podremos lograr en estos casos es una memorización.

No vamos a presentar un modelo didáctico elaborado, sino algunas cuestiones, que pueden tener interés, derivadas de los análisis anteriores y de una reflexión sobre nuestra propia práctica docente.
La estructura lógica en la introducción de los conceptos se podría articular en torno a los siguientes tópicos:

- Concepto de energía

- Principio de conservación de la energía

- La degradación de la energía. Aproximación al concepto de entropia

- Algunos problemas energéticos. Fuentes de energía.

En un primer nivel, se realiza previamente una revisión de la fenomenología del calor, sin ocuparse todavía del concepto de calor ni del de temperatura.

\subsection{Concepto de energía}

Somos plenamente conscientes de la complejidad del concepto de energia pero idónde reside esta complejidad? ¿qué concepto de energía debemos enserar? El término energía ha pasado a formar parte del acervo lingüístico de uso frecuente con lo cual lo encontramos en distintos contextos, con acepciones diferentes, cosa que no siempre contribuye a mejorar la comprensión del mismo. Sin embargo ello influye en el esquema conceptual que forma el alumno y que habrá que considerar a la hora de enseñárselo en la clase de Física.

Además, en un ámbito estrictamente físico su significado es muy general. Aparece en manifestaciones muy diversas, asociadas a una amplia gama de fenómenos (desplazamientos, electricidad, reacciones químicas, radiación...) y si bien considerada globalmente siempre se conserva, en ocasiones se degrada.

El concepto de energía es uno de los más abstractos de los estudiados en un currículo de Física y Química. Su definición por reducción a otros términos más sencillos no es fácil ya que está relacionada con conceptos igualmente conflictivos y abstractos. Todo ello hace que estemos frente a un concepto de índole tal que nos obligue a una introducción distinta a la tan frecuente «definición operativa».

Las características del concepto de energia obligan a abordarlo desde diversas perspectivas, teniendo en cuenta cuál es el punto de partida conceptual de los alumnos e incluyendo el maximo número de situaciones en las que se pueda poner de manifiesto su potencial explicativo. La incorporación de un concepto a la estructura cognoscitiva está relacionada con su capacidad para explicar situaciones, tanto nuevas como aquellas que ya preocupan al alumno.

Podemos partir, por tanto, de una recopilación de material, aportado por los propios alumnos, consistente en textos breves en los que se haga referencia a la energía, obtenidos de la prensa, televisión, o inventados por eilos mismos pero que recojan sus propias ideas (posiblemente algunos recordarán la defínición de energía como capacidad para realizar trabajo, en este caso, podremos poner de manifiesto que esta definición no comporta ningún concepto, sino que se repite de forma mimética). Esto permitirá abrir perspectivas respecto a la 
generalidad del término y por tanto a la riqueza del concepto, a la vez que la imposibilidad de «comprimirio» en una definición breve y concisa. Algo parecido sucede con el concepto de tiempo y en ninguno de los dos casos la dificultad de definición limita su validez.

Intentemos caracterizar el concepto planteando diver * sas situaciones, ahora más "típicamente físicas" (un cuerpo que cae, otro que se desplaza por una superficie horizontal y se para, un muelle que se estira, una bombilla encendida, etc.,) para cuestionar lo que sucede a la energla en cada caso. Al fínal podremos llegar a la idea de que la energía es una característica del sistema, que tiene un valor en cada instante y que se puede modificar cuando el sistema se tranforma.

Podemos plantear ahora el problema de evaluar la cantidad de energia de que dispone un sistema en un instante y situación y la forma en que esta energfa se puede transferir. Es necesario clarificar términos de uso frecuente como: sistema, transformación, estado, proceso, a los que se hace referencia constante y que, sin embargo, suelen permanecer enmascarados en muchas ocasiones.

Los sistemas se transforman por interacción con otros sistemas. Siempre hay un agente dinámico de la transformación y un cambio en la configuración del sistema. La interacción (fuerza) produce una modificación de la disposición inicial del sistema y la magnitud de la transformación se identifica con el trabajo, cuya definición operativa se puede introducir, asl como la idea de que esta definición general es susceptible de adaptación a distintas situaciones (compresión de un gas, electricidad...), para incluir magnitudes físicas de uso más cómodo, adaptadas a los datos más habítuales en cada sistema.

Pero además, los sistemas pueden modificar su energía al interactuar con otro sistema que está a distinta temperatura.

Así pues, la energia es una propiedad del sistema que se manifiesta de muchas formas y que puede variar por la intervención del trabajo $\mathrm{y} / \mathrm{o}$ del calor.

La discusión de algunos ejemplos, a nivel cualitativo, en los que haya sistemas que transformen su energla de una forma en otra, que varien su energia total por la intervención de trabajo, calor, etc., pueden contribuir a clarificar los conceptos. Asi mismo se puede introducir la idea de potencia.

\subsection{Profundizando en el estudio de las transfor- maciones}

Empezamos limitando nuestro estudio a las transformaciones que ya se han estudiado en temas anteriores: cambios de posición y de velocidad. Realizamos un análisis, referido a un cuerpo, de cada una de ellas, introduciendo la energía cinética y potencial según el esque- ma tradicional de identificación de la variación de energfa con el trabajo realizado para lograrlo, que ahora los alumnos están en condiciones de entender. Tiene interés comentar que, los alumnos tienden a confundir el valor de la energia de un sistema en un instante y situación con la variación de energia $y$, en nuestra opinion, esto es indicativo de que el concepto de energía, unido al de la forma de modificar su valor, no están incorporados a la estructura cognoscitiva. La introducción de la energía potencial asociada a las fuerzas conservativas no presenta dificultades especiales siempre que los alumnos estén familiarizados con la fuerza peso (conservativa) y rozamiento (no conservativa).

¿Y si consideramos las dos transformaciones simultáneamente? Al estudiar la calda libre, por ejemplo, podemos deducir que los valores de la energía cinética y potencial varian en cada punto, pero su suma permanece constante, mientras que si consideramos un cuerpo desplazándose por un plano inclinado bajo la acción de una fuerza aplicada (o bien consideramos el razonamiento) varian los valores de la energía cinética, potencial y de la suma de ambas, pero la igualdad entre variación de energia y trabajo realizado se mantiene.

La generalización de los resultados anteriores nos permitirá introducir el principio de conservación de la energia, matizando las dos situaciones: sistemas «conservativos» $y$ «no conservativos».

Estamos ya en condiciones de aproximarnos a otra nueva situación, aquella en la que el sistema intercambia calor. Hasta ahora nos hemos referido a modificaciones del sistema que se detectan macroscópicamente sin ocuparnos de lo que sucedia a nivel microscópico en el sistema. Aceptemos que el sistema permanece en reposo en una determinada posición, por tanto que no varía su energía potencial ni cinética respecto de un sistema de referencia exterior. Si suponemos que el sistema es un recipiente que contiene un gas, fácilmente aceptaremos que las moléculas del mismo se encuen. tran en un estado de agitación permanente al que podemos asociar una energía potencial y cinética. A la suma de esta energía cinética y potencial le denominaremos energia interna y se modifica cuando el sistema intercambia calor. Se introduce en este punto la interpretación de la temperatura y el calor a escala microscópica. Pero la energia interna también puede variar si se realiza trabajo. Estas consideraciones conducen al enunciado de la primera ley de la Termodinámica. Cabe aquí también, la introducción del equivalente me cánico del calor.

La introducción del concepto de calor en este contexto contribuye a erradicar la concepción errónea, sustentada por un amplio número de alumnos, del calor como "fluido", como algo que los cuerpos tienen y pueden aumentar o disminuir según que «ganen» o «pierdan" calor. 


\subsection{Aproximación al concepto de entropía}

Tras el análisis de algunos procesos espontáneos (expansión de un gas, aumento de temperatura de un cuerpo puesto en contacto con otro inicialmente a mayor temperatura, concentración-dilución de una disolución, deslizamiento de un bloque sobre una superficie con rozamiento), conviene llamar la atención sobre el hecho de que los procesos se producen de forma espontánea siempre en un sentido y no en el contrario. Tanto en sentido directo como inverso los sistemas cumplen la primera ley de la Termodinánica. ¿Por qué nunca observamos el proceso inverso?

El proceso en sentido contrario implicaría una ordenación en el movimiento aleatorio de las moléculas, significaria la conversión de energía interna en trabajo. Tomando el ejemplo del bloque que desliza, significaría que la energía empleada en aumentar la temperatura de la superficie de contacto al deslizar (que es el proceso al que nos referimos), se recupera, de forma que a medida que la superficie alcanza su temperatura inicial, el bloque se mueve en sentido contrario, dirigiéndose a su posición primitiva. Evidentemente esto no sucede en realidad. La explicación implica la introducción de una magnitud característica del estado del sistema, denominada entropfa, asociada al desorden del mismo y caracterizada porque en todos los procesos espontáneos la entropía del universo aumenta. La asociación entre procesos espontáneos y aumento de entropía es $\tan$ sólida que muchas veces se dice que el aumento de entropía indica el sentido de la flecha del tiempo.

En estos procesos se conserva la energía, pero el aumernto de entropia que los acompaña explica que no se restablezca espontáneamente la situación inicial y por tanto que no pueda recuperar toda la energía. Parte de la energia se «invierte» en aumentar la entropía y esta parte no es recuperable, no se podrá utilizar para realizar trabajo.

\section{COMENTARIO FINAL}

El esquema propuesto permite el desarrollo de curricula circulares, en los que se seleccionen los contenidos según su complejidad (por ejemplo, se puede introducir el concepto de entropia sólo en niveles superiores), pero el esquema conceptual, propiamente dicho, se empieza a construir desde el principio, de forma que otros conceptos se incorporen de manera natural. De este modo no se favorece la persistencia de errores conceptuales (concepto de calor) como sucede con otros planteamientos. El trabajo se completa con la discusión de diferentes fuentes de energla, el nivel de profundidad que se desee, pudiendo incluso, realizar una interesante actividad interdisciplinar. $\mathrm{La}$ incorporación de problemas como el de la energía of rece la posibilidad de relacionar la escuela con la problemática cotidiana de la sociedad en que vive el alumno.

Se podria criticar la introducción de conceptos termodinámicos argumentando que éstos son excesivamente complicados para los alumnos de enseñanza media. El nivel de desarrollo de la estructura mental requerido para acceder a la comprensión de estos conceptos, o de la teoría cinética-molecular no es superior al necesario para entender los principios de Newton (Shayer y Adey, 1984), cuya incorporación al curriculum no se cuestiona. Corresponde al nivel operacional formal, que es el nivel que deberfa poseer el alumno cuando menos al final de la ensenanza media.

La modificación de estructura propuesta no significa en realidad ampliación de temario. Aunque el problema de la extensión del temario sólo se resolverá, desde nuestro punto de vista, cuando se realice una selección racional de contenidos desde la enseffanza básica hasta la Universidad, considerando no sólo la propia 16gica interna de la Ciencia que se pretende enseflar, sino el nivel de desarrollo intelectual requerido para su aprendizaje y el real de los alumnos a los que se dirige. Olvidar cualquiera de estos aspectos conducirá, una vez más, a la sensación de fracaso que con demasiada frecuencia acompana nuestra tarea.

\section{REFERENCIAS BIBLIOGRAFICAS}

ANDERSON, C.E., BOTTINELLI, CH.A., 1981, Something special for teachers. A school house energy teaching program. SEED: Schoolhouse energy efficiency demostration, (Tenneco, Inc., Houston, Tex.).

BLISS, J., OGBORN, J., 1985, Children's choises of uses of energy, European Journal of Science Education, Vol. 7, pp. 195-203.
CANIPE, S.L., 1982, Typing into computers, Paper presented al the energy, environment and economics teacher workshop. (Geographic source US, Nort Carolina).

COHEN, M.R., 1981, «Problem solving as a goal of energy and environmental education", Journal of Environmental Education, Vol. 13, pp. 17-21. 
DUIT, R., 1984, Learning the energy concep in school empirical results from the Philippines and West Germany, Physics Education, Vol. 19, pp. 59-66.

DYKSTRA, D.I., 1982, A learning cycle on exponential growth and the energy crises, Physics Teacher, Vol. 20, pp. $245-46$.

FLAVELL, J.H., 1984, El desarrollo cognitivo, (Visor: Madrid; Prentice Hall: New Jersey, 1977).

GORE, P. and others, 1980, Theaching energy awareness. Environmental education series, (Center for Teaching International Relations; Denver Univ., Colorado).

HABER-SCHAIM, U., 1983, The role of the second law of thermodynamics in energy education, 1983, The Physics Teacher, Vol. 21, pp. 17-20.

HAGGIS, S.M., 1981, The environment and energy as integrating themes in Science Education, Environmental Education and Information, Vol. 1, pp. 225-31.

HICKS, N., 1983, Energy is the capacity to do work or is it?. The Physics Teacher, Vol. 21, pp. 529-30.

INHELDER, B., PIAGET, J., 1972, De la lógica del niño a la logica del adolescente, (Paidos, Buenos Aires; Press Univ, de France 1955).

JONES; C., 198I, Alternative Energy Proyects in Schools, School Science Review, Vol. 63, pp. 260-66.
LEWIS, J.L., 1981, Energy education and environment in Science Curriculum, Environmental Education and Information, Vol. 1, pp. 189-96.

SELL, N.J., VAN KOEVERING, TH.E., 1981, The energyenvironment simulator as a classroom aid, Journal of Computers in Mathematics and Science Teaching, Vol. 1, pp. 20-22.

SHAYER, M., ADEY, PH., 1984, La Ciencia de enseffar Ciencias, (Narcea, Madrid, Heinemann Educational Books, Londres, 1981).

WARD, A., 1983, Approaching an elementary concep of energy: Part two, School Science Review, Vol. 65, pp. 230-32.

WARPINSKI, R., 1984, Environmental Education: Back to Basic, Nature Study, Vol. 37, pp. 14-16.

WATTS, M., 1983, Some alternative views of energy, Physics Education, Vol. 18 pp. 213-17.

WHITE, J.A., FOWLER, J.M., 1983, Energy education in the Schools. Reswlts of a survey of the penetration of energy education into the classroom, (National Science Teachers Association, Washington, D.C.).

WILSON, M.A., YASI, E.E., 1980, Developing energy education curriculum, (Hampshire educational Collaborative: Northampton. Massachusetts. U.S.). 